\title{
Near-infrared photothermal therapy using anti-EGFR-gold nanorod conjugates for triple negative breast cancer
}

\author{
Meihua Zhang ${ }^{1,2,3, *}$, Hoe Suk Kim ${ }^{1, *}$, Tiefeng Jin ${ }^{1,4}$, Jisu Woo ${ }^{1}$, Yin Ji Piao ${ }^{1,2}$ and Woo \\ Kyung Moon ${ }^{1,2}$ \\ ${ }^{1}$ Department of Radiology, Seoul National University Hospital, Jongno-gu, Seoul 03080, Korea \\ ${ }^{2}$ Department of Biomedical Sciences, Seoul National University College of Medicine, Jongno-gu, Seoul 110-799, Korea \\ ${ }^{3}$ Department of Radiology, Yanbian University Hospital, JiLin Province 133000, China \\ ${ }^{4}$ Department of Pathology and Cancer Research Center, Yanbian University Medical College, Yanji 133002, China \\ *These authors have contributed equally to this work \\ Correspondence to: Woo Kyung Moon, email: moonwk@snu.ac.kr
}

Keywords: near-infrared photothermal therapy, photoacoustic imaging, breast cancer, gold nanorod, epidermal growth factor receptor

Received: May 14, $2017 \quad$ Accepted: August 17, $2017 \quad$ Published: September 23, 2017

Copyright: Zhang et al. This is an open-access article distributed under the terms of the Creative Commons Attribution License 3.0 (CC BY 3.0), which permits unrestricted use, distribution, and reproduction in any medium, provided the original author and source are credited.

\section{ABSTRACT}

Current EGFR-targeted therapy for triple negative breast cancer (TNBC) has produced disappointing results. A rational therapeutic strategy to improve EGFRtargeted treatment for TNBC is therefore needed. In this study we evaluated the feasibility of treating TNBC using photoacoustic imaging (PAI)-guided near-infrared photothermal therapy (NIR-PTT) with anti-EGFR-conjugated gold nanorods (antiEGFR-GN). NIR-PTT combined with anti-EGFR-GN exerted synergistic anti-proliferative and apoptotic actions through upregulation of HSP70 and cleaved caspase-3, downregulation of Ki-67 and EGFR, and inhibition of several intracellular signaling molecules (mTOR, AKT, ERK1/2 and FAK). These combined effects give this approach significant efficacy. Our findings suggest PAI-guided NIR-PTT using anti-EGFR-GN represent a novel and effective strategy for EGFR-targeted therapy in TNBC.

\section{INTRODUCTION}

Although triple negative breast cancer (TNBC) is a small percentage of all breast cancers, TNBC is one of the most challenging types of breast cancer for basic and clinical research because TNBC patients display a high risk of relapse, shorter overall survival and limited therapeutic options after completion of conventional chemotherapy compared with patients with other breast cancer subtypes $[1,2]$. Because of the lack of a well-defined clinical therapy strategy, advances in the design of strategies for the treatment of TNBC require further elucidation, by combined targeted therapy, of the molecular mechanisms underlying TNBC genotypic and phenotypic heterogeneity. Approximately 70 to $80 \%$ of TNBCs more frequently overexpress epidermal growth factor receptor (EGFR), which is emerging as a therapeutic target $[3,4]$. Many clinical studies on TNBC patients using an EGFR antibody (cetuximab) and EGFR pathway inhibitors (lapatinib and gefitinib) have been evaluated, yet EGFR-targeted therapy has produced a response in only a minority of TNBC patients $[5,6]$. A rational therapeutic strategy to overcome the limitations of current EGFR-targeted treatments of TNBC patients is absolutely required for future clinical trials.

Non-invasive monitoring of tumors in vivo with biocompatible contrast agents including specific targeting molecules after multiple rounds of intravenous administration may be capable of being optimized and an efficacious treatment of cancer. Photoacoustic imaging (PAI), which can create multi-contrast images of living biological structures ranging from organelles to organs, 
has shown great translational potential from bench to bedside due to its inexpensiveness and convenience for combination with clinical ultrasound (US) [7]. Gold nanorods (GN) conjugated with specific molecules including antibodies has been proposed as an attractive PAI contrast agent for allowing the GN to bind selectively to certain primary tumors and metastatic sites in vivo [8-10]. GN with particularly near-infrared (NIR) optical properties at wavelengths from 700 to $1000 \mathrm{~nm}$, where NIR radiation is able to penetrate the skin without damaging normal tissues, has revealed great potential for simultaneously combining selective targeted imaging and NIR-mediated photothermal therapy (NIR-PTT) in diverse types of cancer [11-14]. In recent years, NIR-PTT using GN embedded within tumors can cause apoptotic or necrotic damage to tumor cells by inducing a localized hyperthermia effect, suggesting a promising therapeutic technique with great potential for cancer treatment due to its minimal invasiveness and high spatial selectivity [1521]. To date, GN heated with an NIR light has improved the efficiency and safety of therapy against various solid tumors, yet few studies have demonstrated the successful treatment of TNBC.

We previously applied US and PAI using GN conjugated with anti-EGFR antibody (anti-EGFR-GN) for the selective visualization of EGFR-positive TNBCs and demonstrated that US-guided PAI can sensitively detect solid primary tumors as well as lymph node (LN) micrometastases in xenograft mice intravenously injected with anti-EGFR-GN [22]. Anti-EGFR-GN with a desired NIR wavelength (approximately $808 \mathrm{~nm}$ ) is an ideal contrast agent for application of both cancer cell imaging and NIR-PTT. In the present study, we evaluated the feasibility of using anti-EGFR-GN combined with NIRPTT for more effective EGFR-targeted therapy of TNBCs with the help of non-invasive monitoring of selective targeting as well as therapeutic response using US and PAI.

\section{RESULTS}

\section{Anti-EGFR-GN selectively uptaken by EGFR- overexpressing cells, and the combination of anti-EGFR-GN and NIR-PTT synergistically induces cell death}

High EGFR expression was detected in TNBC cell lines (Hs578T, HCC-38, MDA-MB-468 and MDAMB-231), but not in the other subtype cell lines (MCF7 and BT474) (Figure 1A). Immunocytochemistry also indicated high EGFR expression in MDA-MB-231 cells (Figure 1B). Silver staining showed that the uptake of anti-EGFR-GN by MDA-MB-231 cells overexpressing EGFR was inhibited by competition with free antiEGFR antibody, indicating the specificity of EGFRtargeting (Supplementary Figure 1). Consistent with the understanding that nanoparticles are endocytosed by cells [10], TEM images revealed a large amount of anti-EGFRGN in the endosomes or lysosomes of MDA-MB-231 cells. Whereas, low cytoplasmic GN was rarely observed (Figure 1C). $24 \mathrm{~h}$ or $72 \mathrm{~h}$ treatment with anti-EGFR-GN ( $208.50 \pm 6.78 \%$ or $364.31 \pm 11.13 \%$, respectively) or antiEGFR $(207.53 \pm 1.48 \%$ or $339.20 \pm 17.14 \%$, respectively) significantly inhibited the cell growth versus untreated control $(P<0.01$ or $P<0.001)$, but GN did not affect the cell growth (Figure 1D). Additionally, we observed an anti-cancer effect of anti-EGFR-GNs in another TNBC cell line, MDA-MB-468. Anti-EGFR-GNs suppressed the growth of MDA-MB-468 cells and exhibited similar anti-cancer efficacies observed in MDA-MB-231 cells (Supplementary Figure 2). In vitro NIR-PTT (1.5 W/ $\mathrm{cm}^{2}$, for $3 \mathrm{~min}$ ) with anti-EGFR-GN can elevate the temperature of cell culture media from to $39^{\circ} \mathrm{C}$ to $43^{\circ} \mathrm{C}$. In flow cytometric analysis of annexin $\mathrm{V} /$ propidium iodide (PI) (Figure 1E), a significant apoptotic cell death was not detected in groups treated with NIR-PTT $(2.66 \pm 0.11 \%)$, GN $(3.91 \pm 0.17 \%)$, and GN+NIR-PTT $(4.46 \pm 0.16 \%)$ compared with control $(1.74 \pm 0.12 \%)$. However, treatment with anti-EGFR (20.10 $\pm 0.81 \%)$, anti-EGFR-GN $(27.81 \pm 1.75 \%)$, a combination of antiEGFR and NIR-PTT $(26.46 \pm 1.16 \%)$ and a combination of anti-EGFR-GN and NIR-PTT (41.51 $\pm 0.54 \%)$ induced significant apoptotic cell death $(P<0.001)$. The combined anti-EGFR-GN and NIR-PTT led to an increase in therapeutic temperature to $43^{\circ} \mathrm{C}$, consequently resulting in the most effective apoptotic death.

\section{Anti-EGFR-GN combined with NIR-PTT augmented anti-proliferative and cell death signaling}

In this study, GN and GN+NIR-PTT groups were excluded because the cellular uptake of GN and GN+NIR-PTT-induced apoptosis was not observed in MDA-MB-231 cells. We next investigated the effect of anti-EGFR, anti-EGFR-GN and anti-EGFR-GN+NIRPTT on the alteration of intracellular proteins involved in proliferative and apoptotic activity. As shown in Figure 2A and 2B, there was no difference in HSP90 levels among all groups. Whereas, induction of HSP70 was obvious in groups treated with anti-EGFR (151.53 $\pm 15.59 \%)$, antiEGFR-GN (520.43 $\pm 90.87 \%)$ and anti-EGFR-GN+NIRPTT $(782.14 \pm 80.74 \%)$ relative to control $(P<0.001)$. The proliferation marker Ki-67 was significantly decreased in groups treated with anti-EGFR $(17.1 \pm 1.37 \%)$, antiEGFR-GN $(9.06 \pm 0.60 \%)$, and anti-EGFR-GN+NIR-PTT $(6.19 \pm 2.5 \%)$ relative to control $(P<0.001)$. The apoptotic marker cleaved caspase-3 was significantly increased in groups treated with anti-EGFR (141.11 $\pm 11.10 \%)$, anti-EGFR-GN $\quad(259.08 \pm 17.23 \%)$, and anti-EGFRGN+NIR-PTT $(422.33 \pm 61.36 \%)$ relative to control. The other apoptotic biomarker, cleaved poly(ADP-ribose) 
polymerase-1 (PARP-1) was also evaluated. Cells treated with anti-EGFR-GN+NIR-PTT, although an accumulation of cleaved PARP-1 was not significant as cleaved caspase- 3 result, expressed comparable high cleaved PARP-1 $(218.10 \pm 49.31 \%)$ as compared with those of NIR-PTT, GN, GN+NIR-PTT and anti-EGFRGN (Supplementary Figure 3). A significant decrease of EGFR was evident in the groups treated with antiEGFR-GN (16.49 $\pm 4.64 \%)$ and anti-EGFR-GN+NIRPTT $(5.89 \pm 1.16 \%)$ relative to control $(P<0.001)$. In the experimental condition for short-term treatment $(1,5$, $15,30 \mathrm{~min}$ ) with GN or anti-EGFR-GN, the treatment with GN alone increased the phosphorylations of AKT and ERK1/2, but the treatment with anti-EGFR-GN alone suppressed the phosphorylations of FAK and AKT (Supplementary Figure 4). As shown in Figure $2 \mathrm{C}$ and 2D, phosphorylations of mTOR, FAK and ERK1/2 were significantly decreased in the groups treated with NIRPTT $(43.99 \pm 4.94 \%, 36.54 \pm 1.37 \%$ and $50.97 \pm 2.62 \%$, respectively), anti-EGFR $(43.46 \pm 6.43 \%, 33.60 \pm 2.59 \%$ and $22.64 \pm 2.79 \%$, respectively), anti-EGFR-GN $(28.51 \pm 4.74 \%, \quad 36.34 \pm 2.11 \%$ and $23.04 \pm 4.44 \%$, respectively) and anti-EGFR-GN+NIR-PTT $(8.51 \pm 1.11 \%$, $27.67 \pm 0.87 \%$ and $6.37 \pm 1.26 \%$, respectively) relative to control $(P<0.001)$. Phosphorylated AKT was significantly decreased in the groups treated with anti-EGFR $(68.12 \pm 5.89 \%, P<0.01)$, anti-EGFR-GN $(18.83 \pm 1.26 \%$, $P<0.001)$ and anti-EGFR-GN+NIR-PTT $(11.48 \pm 2.78 \%$, $P<0.001)$ relative to control. Anti-EGFR-GN+NIR-PTT exerted the most potent synergistic, anti-proliferative and cell death effect through the downregulation of Ki67 and EGFR, the inhibition of mTOR, FAK, AKT and ERK1/2-mediated intracellular signaling pathways and the activation of caspase- 3 .

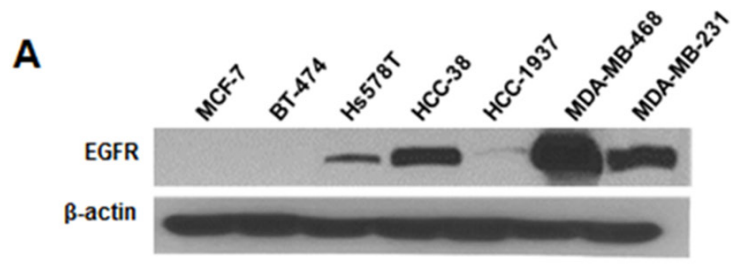

B

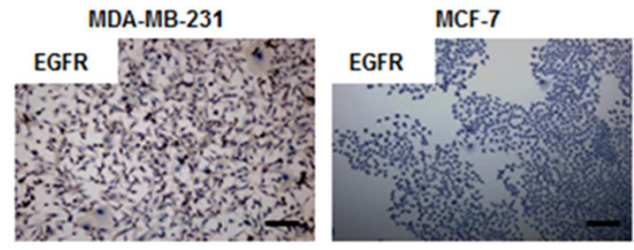

C
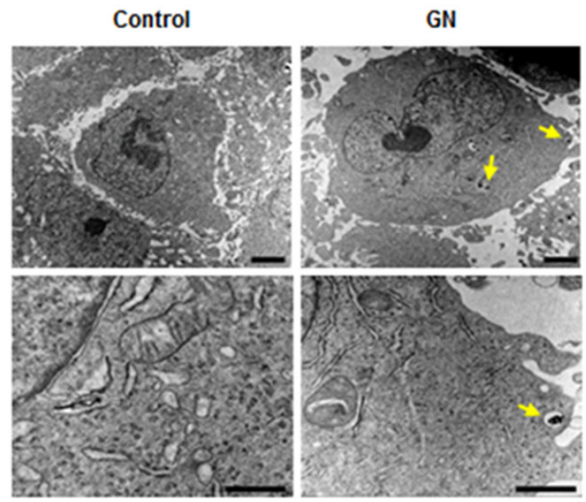

anti-EGFR-GN

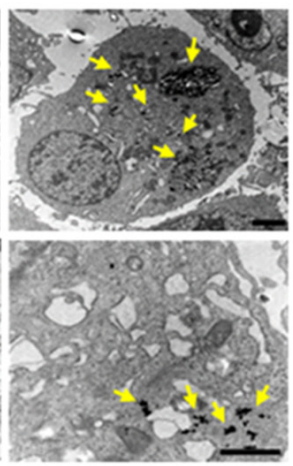

D

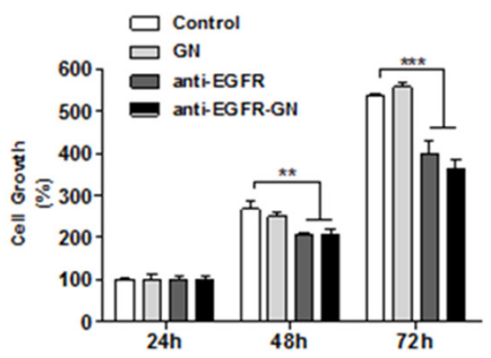

E

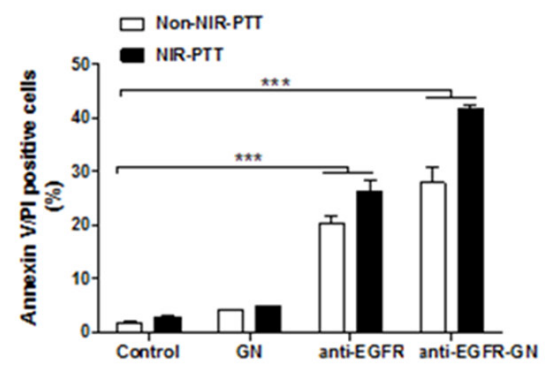

Figure 1: Analysis of selective uptake of anti-EGFR-GN, anti-proliferative and apoptotic action caused by anti-EGFRGN combined NIR-PTT. (A) Western blot of EGFR in the total lysates of TNBC cell lines (Hs578T, HCC-38, HCC-1937, MDAMB-468, MDA-MB-231), ER+ cell line (MCF-7) and HER2+ cell line (BT-474). (B) Immunocytochemistry for EGFR in MDA-MB-231 cells and MCF-7 cells. Scale bar: $300 \mu \mathrm{m}$. (C) TEM images of MDA-MB-231 cells treated with $1.84 \mu \mathrm{g} / \mathrm{ml}$ of GN or anti-EGFR-GN (endocytic uptake, yellow arrows) for $24 \mathrm{~h}$. Scale bar: $1 \mu \mathrm{m}$. (D) Analysis of cell growth (mean \pm S.E., $\mathrm{n}=5$ ) assessed by MTT assay in MDAMB-231 cells treated with $1.84 \mu \mathrm{g} / \mathrm{ml}$ of GN and anti-EGFR-GN or anti-EGFR antibody $(0.22 \mu \mathrm{g} / \mathrm{ml})$ for 24, 48 and $72 \mathrm{~h}$. (E) Apoptotic cell death (mean \pm S.E., $\mathrm{n}=4$ ) assessed by flow cytometric analysis of annexin $\mathrm{V}$ and PI in MDA-MB-231 cells treated with $\mathrm{GN}(1.84 \mu \mathrm{g} / \mathrm{ml})$ and anti-EGFR-GN ( $1.84 \mu \mathrm{g} / \mathrm{ml} \mathrm{GN}, 0.22 \mu \mathrm{g} / \mathrm{ml}$ anti-EGFR antibody) or anti-EGFR antibody $(0.22 \mu \mathrm{g} / \mathrm{ml})$ for $24 \mathrm{~h}$ and subsequent NIR-PTT for 3 min. $* P<0.05, * * P<0.01, * * * P<0.001$. 


\section{Anti-EGFR-GN combined with NIR-PTT was a more effective tumor treatment than anti-EGFR- antibody therapy}

The timing of NIR-PTT treatment at which intravenously injected anti-EGFR-GNs maximally accumulated into the tumors of mice using serial followup PAI was determined based on our previous study [22]. As shown in Figure 3A, there was no different in PA signal amplitude of GN- and anti-EGFR-GN-treated MCF-7 tumors at each time point. The maximal value of the anti-EGFR-GN-enhanced PA signals $(9.91 \pm 0.41$ $\mathrm{AU}$ and $8.59 \pm 0.17 \mathrm{AU}$, respectively) were reached 24 and $48 \mathrm{~h}$ post-injection in MDA-MB-231 tumors and was significantly higher (approximately 2-fold) than GN-enhanced PA signals (5.97 $\pm 0.12 \mathrm{AU}$ and $3.84 \pm 0.09$ AU, respectively) (Figure 3B, $P<0.001$ ). GN-enhanced PA signals were decreased into a basal level after $48 \mathrm{~h}$. Figure 3B shows the representative US-guided PAI of MCF-7 and MDA-MB-231 tumors before and at $48 \mathrm{~h}$ after intravenous injection with GN or anti-EGFR-GN. Silver staining revealed nanoparticle accumulation in only MDA-MB-231 tumor tissues at $48 \mathrm{~h}$ post-injection of antiEGFR-GN (Figure 3C). These results demonstrate that anti-EGFR-GN selectively accumulates into the MDAMB-231 tumors, but GN rapidly clears out of the MCF-7 and MDA-MB-231 tumors. In order to effectively achieve
EGFR-targeted NIR-PTT for MDA-MB-231 tumor treatment, we excluded the GN+NIR-PTT group from the in vivo study and selected an NIR-PTT treatment time of $48 \mathrm{~h}$ after anti-EGFR-GN injection. Irradiation with NIR light for $3 \mathrm{~min}$ induced mild hyperthermia $\left(43^{\circ} \mathrm{C}\right)$ in tumor skin. The longitudinal follow-up US-guided PAI and BLI were acquired from MDA-MB-231 tumors before and after NIR-PTT in each group (Figure 3D and 3E). In the anti-EGFR-GN+NIR-PTT group, the PA signal amplitude and bioluminescence activity of tumors remarkably decreased after second and third treatments, which may selectively reflect EGFR-positive tumor cell damage or tumor vascular insult. Continuous decrease of tumor volumes was observed in the anti-EGFR-, anti-EGFR-GNand anti-EGFR-GN+NIR-PTT-treated mice versus control (Figure 3F). Anti-EGFR-GN+NIR-PTT resulted in almost complete tumor regression. Treatment with anti-EGFR, anti-EGFR-GN and anti-EGFR-GN+NIT-PTT did not cause significant differences in the body weights of mice and the damage of normal tissues compared to those of untreated mice, suggesting the absence of physical distress over the course of the experiment.

The gross images of tumor tissues revealed that the smallest residual tumor volume was in the anti-EGFRGN+NIR-PTT group (Figures 4A and 4B). Consistent with the in vitro results of cultured cells, immunohistochemical analysis showed much higher caspase-3 $(73.33 \pm 6.17 \%)$
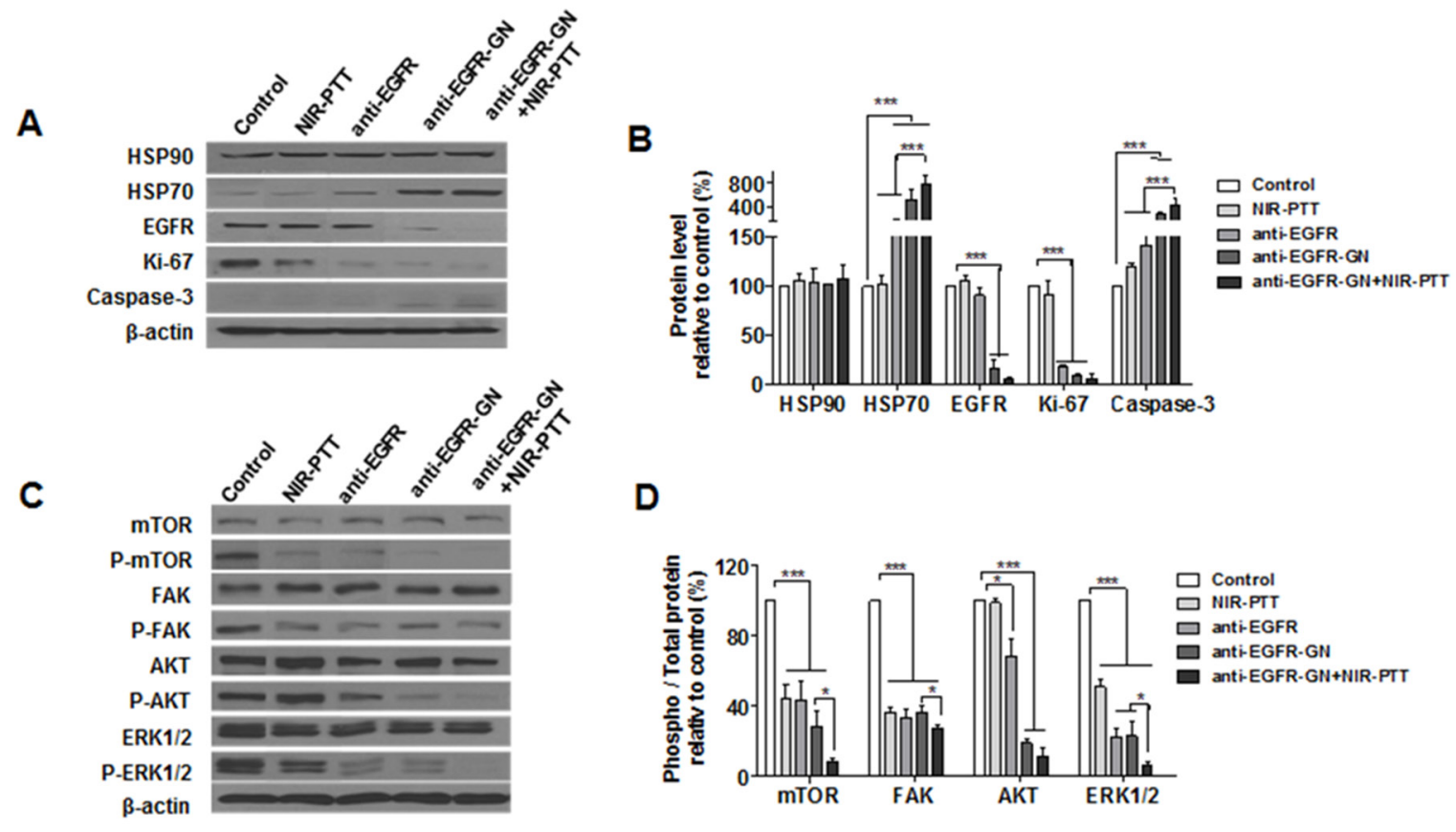

Figure 2: Analysis of the fundamental mechanisms of anti-proliferation and apoptosis triggered by anti-EGFR-GN combined with NIR-PTT. (A and C) Western blot of heat shock proteins (HSP90, HSP70), EGFR, Ki-67, cleaved caspase-3 and EGFRmediated intracellular signaling molecules (mTOR, FAK, AKT, ERK1/2) in MDA-MB-231 cells treated with GN (1.84 $\mu \mathrm{g} / \mathrm{ml})$, anti-EGFRGN $(1.84 \mu \mathrm{g} / \mathrm{ml} \mathrm{GN}, 0.22 \mu \mathrm{g} / \mathrm{ml}$ anti-EGFR-antibody) and anti-EGFR antibody $(0.22 \mu \mathrm{g} / \mathrm{ml})$ for $24 \mathrm{~h}$ and subsequent NIR-PTT for 3 min. (B and D) Data (mean \pm S.E., $\mathrm{n}=3$ ) obtained from western blot experiments. $* * P<0.01, * * * P<0.001$. 
and TUNEL levels $(5.2 \pm 0.38 \%)$ and remarkably lower $\mathrm{Ki}-67(3.67 \pm 0.88 \%)$ and EGFR $(0.83 \pm 0.20 \%)$ levels in the anti-EGFR-GN+NIR-PTT tumors compared with the other tumors (Figures 4C and 4D, $P<0.001$ ). This indicates that anti-EGFR-GN+NIR-PTT led to the most effective therapy for tumor regression through augmentation of the synergic mechanisms of anti-proliferation and apoptosis. Silver staining revealed very little anti-EGFR-GN accumulation in the kidney at 4 days after the third posttreatment of anti-EGFR-GN+NIR-PTT. H\&E staining showed no damage or histological alteration of the liver, spleen or kidney of each group (Figure 4E), implying that anti-EGFR-GN was eventually cleared out of body without toxicity.

\section{DISCUSSION}

We here demonstrate that anti-EGFR-GNs is a useful therapeutic strategy for applying EGFR-targeted
NIR-PTT to aggressive TNBC due to their properties of selective tumor cell targeting, long circulation time and desired optical absorption upon NIR irradiation. When combining NIR-PTT with anti-EGFR-GNs in EGFRpositive MDA-MB-231 cells and tumors, we observed obstruction of the EGFR-mediated intracellular signal pathway (low phosphorylated mTOR, AKT, ERK1/2 and FAK levels) involved in cell proliferation and survival, as well as strong anti-proliferative (low Ki-67 level) and apoptotic activity (high cleaved caspase- 3 and TUNEL levels).

TNBC is a biologically heterogeneous disease. Translational and clinical studies for emerging targeted TNBC therapies have been facilitated by increased understanding of the aberrant gene expression regulating growth and survival $[3,4]$. EGFR represents a clinically relevant molecular target for TNBC patients [6, 23, 24]. Many clinical Phase I/II investigations for TNBC patients have tried using Cetuximab, FDA-approved anti-EGFR
A

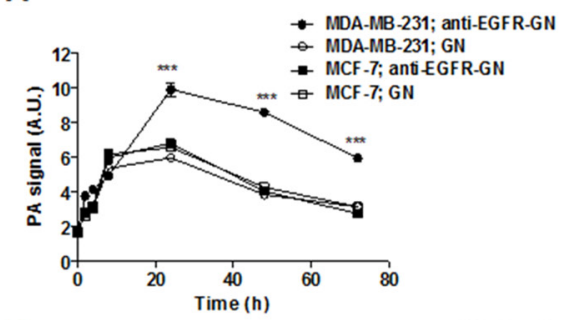

D

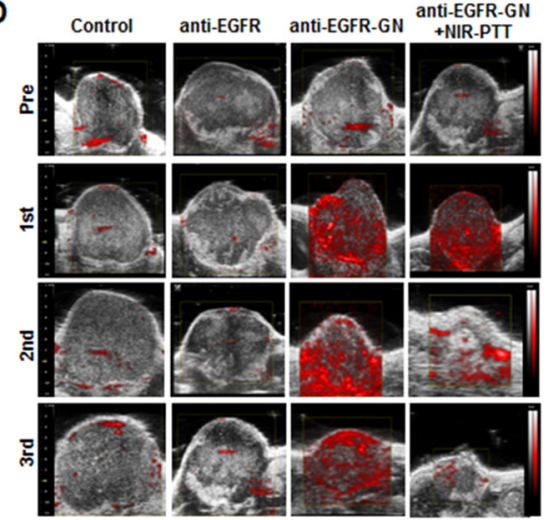

B

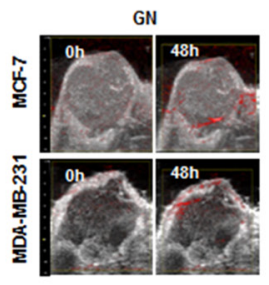

anti-EGFR-GN

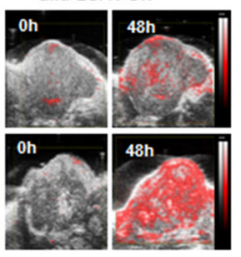

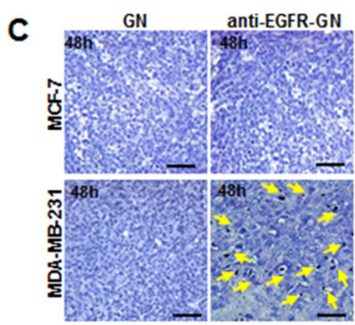

anti-EGFR-GN

E

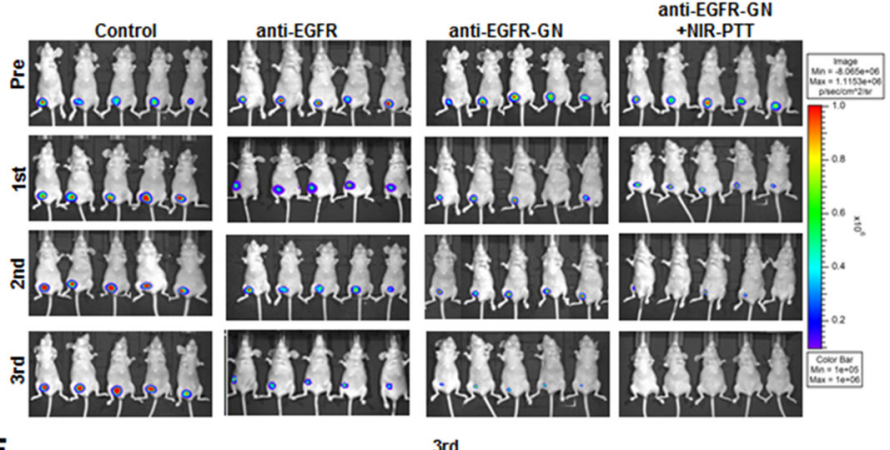

$\mathbf{F}$

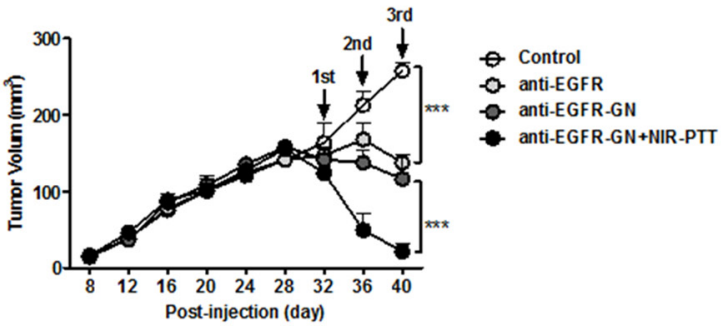

Figure 3: Analysis of PAI-and BLI-guided NIR-PTT combined with anti-EGFR-GN in xenograft tumors. (A) PA signals (mean \pm S.E.) measured from MCF-7 and MDA-MB-231 tumors before and $2 \mathrm{~h}, 4 \mathrm{~h}, 8 \mathrm{~h}, 24 \mathrm{~h}, 48 \mathrm{~h}$ and $72 \mathrm{~h}$ after intravenous injection with of GN $(7.7 \mathrm{mg} / \mathrm{kg})$ or anti-EGFR-GN $(7.7 \mathrm{mg} / \mathrm{kg} \mathrm{GN}, 0.92 \mathrm{mg} / \mathrm{kg}$ anti-EGFR antibody). (B) Representative US and PAI in MCF-7 and MDA-MB-231 tumors before and at $48 \mathrm{~h}$ after injection of GN or anti-EGFR-GN. (C) Silver staining of MCF-7 and MDA-MB-231 tumors isolated from mice at $48 \mathrm{~h}$ post-injection. Yellow arrows indicate the accumulated anti-EGFR-GNs. Scale bar: $200 \mu \mathrm{m}$. (D and E) NIR-PTT was performed at $48 \mathrm{~h}$ after injection of anti-EGFR-GN three times at 3-day intervals. Serial follow-up PAI and BLI of tumors in of each group before and after administration of anti-EGFR antibody $(0.92 \mathrm{mg} / \mathrm{kg})$, anti-EGFR-GN (7.7 mg/kg) and anti-EGFR-GN (7.7 $\mathrm{mg} / \mathrm{kg} \mathrm{GN}, 0.92 \mathrm{mg} / \mathrm{kg}$ anti-EGFR antibody)+NIR-PTT. (F) Tumor volumes (mean \pm S.E.) measured from 5 tumors of each group. $* P<0.05$, $* * P<0.01, * * * P<0.001$. 
antibody [5, 6, 25, 26], yet the results have been somewhat disappointing. With the help of non-invasive longitudinal US-guided PAI, we have previously reported that antiEGFR-GN has great promise in taking advantage of EGFR-targeted diagnosis [22].

NIR-PTT has gained popularity and has quickly developed in recent years due to minimally invasive treatments for cancer therapy in both fundamental and clinical studies. Considerable research is being directed towards developing GN as a theranostic platform [10, $14,27]$. GN has been brought to the forefront of cancer research in recent years due to convenient surface modifications for specific molecular delivery and tunable optical properties for PAI and NIR-PTT applications [7, $10,14,28,29]$. One of the most promising directions in NIR-PTT is the use of GN with 10 to $100 \mathrm{~nm}$ in diameter and peak transmission at approximately $800 \mathrm{~nm}$, owing to its long term circulation from an intravenous injection and its high capability of deeper penetration into tissues [7, 8, 15, 29]. Anti-EGFR-GN is an ideal agent for NIRPTT applications in vivo due to its properties of peak absorbance at $808 \mathrm{~nm}$ as well as their diameter and length of $10 \times 40 \mathrm{~nm}$. However, to the best of our knowledge, NIR-PTT using anti-EGFR-GN has not yet been applied to TNBCs. Moreover, the detailed biological mechanisms of cellular therapeutic response to anti-EGFR-GN combined with NIR-PTT have not been explored.

A water-filtered infrared A lamp (wIRA), a special form of heat radiation with high tissue penetration and low thermal load to the skin surface, has been applied in wound healing without discomfort to the patient and can be used for cancer therapy [30]. The possible modes of cell death triggered by PTT include necrosis and apoptosis. More specifically, high-energy irradiation can lead to necrosis $\left(>45^{\circ} \mathrm{C}\right)$, and low-energy irradiation $\left(<45^{\circ} \mathrm{C}\right)$ can promote apoptosis $[31,32]$. In our study, the
A

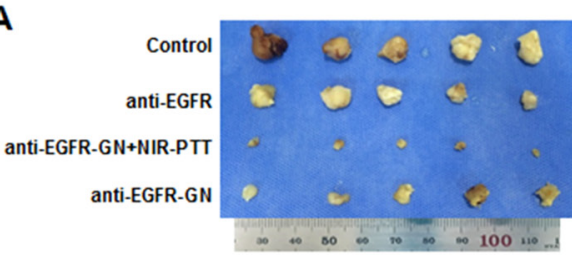

c

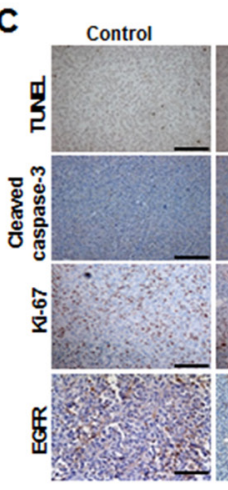

E

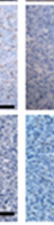

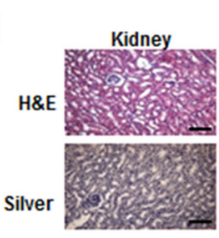

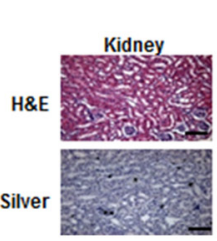

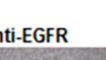

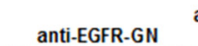
anti-EGFR-GN
+ NIR-PTT
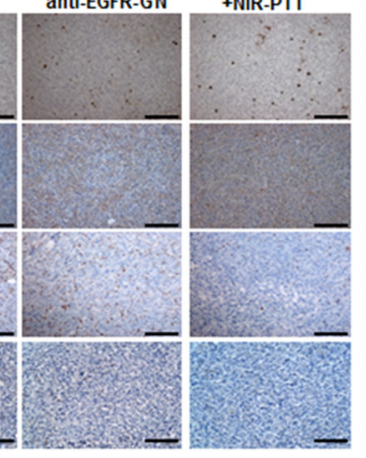
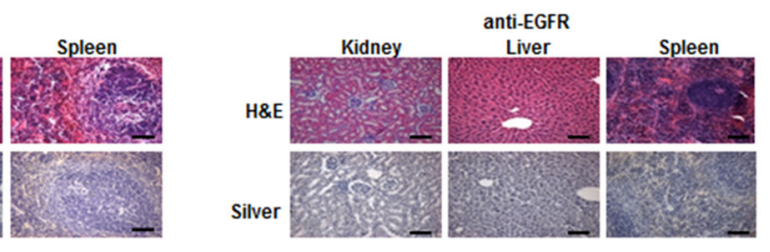
anti-EGFR-GN
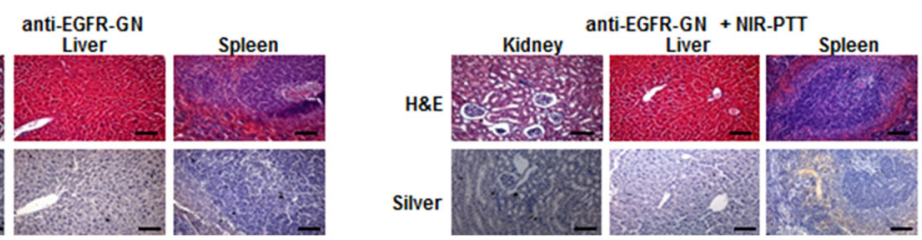

Figure 4: Histological analysis of xenograft tumors. Tumor, kidney, liver and spleen was isolated from mice at 4 days after third treatment of anti-EGFR antibody $(0.92 \mathrm{mg} / \mathrm{kg})$, anti-EGFR-GN $(7.7 \mathrm{mg} / \mathrm{kg} \mathrm{GN}, 0.92 \mathrm{mg} / \mathrm{kg}$ anti-EGFR antibody)+NIR-PTT. (A and B) Gross images and H\&E images of tumors isolated from each mouse group. (C) Immunohistochemistry images of TUNEL, cleaved caspase-3, Ki-67 and EGFR. (D) Quantitative analysis (mean \pm S.E.) of immunohistochemistry obtained from 5 tumor sections of each group. (E) $\mathrm{H} \& \mathrm{E}$ and silver staining of kidney, liver and spleen tissues isolated from each mouse group. Scale bar: $100 \mu \mathrm{m}$. $* P<0.05, * * P$ $<0.01, * * * P<0.001$. 
temperature of culture medium and tumor skin increased to $39-43^{\circ} \mathrm{C}$ after irradiation with wIRA for $3 \mathrm{~min}$.

We found that significant uptake of anti-EGFR-GNs suppressed the proliferative activity of MDA-MB-231 cells compared to that with non-targeted GNs, which is involved in blocking the activation of EGFR-mediated signaling molecules (mTOR, AKT, FAK and ERK1/2) and suppressing EGFR and Ki-67 expressions through the selective and effective delivery of the anti-EGFRantibodies. In our study, anti-EGFR-GNs combined with NIR-PTT induced an increase in HSP70 and cleaved caspase-3, inhibited the phosphorylations of mTOR, AKT, FAK and ERK1/2 and suppressed EGFR and Ki-67 in cultured MDA-MB-231 cells, which suggests a molecular mechanism of synergistic apoptotic and anti-proliferative activity, not necrosis. In addition, our recent study showed that a combination of anti-EGFR-GN and NIR-PTT induced an autophagic cell death mechanism, resulting in the strongest cell death [33]. Taken together in an in vitro study, anti-EGFR-GN combined with NIR-PTT has proven to be a selective and effective therapy in EGFRexpressing TNBC cells. As we expected, anti-EGFR-GNs combined with NIR-PTT led to remarkably greater tumor regression compared to treatment with either anti-EGFR antibody alone or anti-EGFR-GNs alone, significantly reducing proliferation activity (low $\mathrm{Ki}-67$ level) and inducing apoptotic activity (high cleaved caspase-3 and TUNEL levels) in sections of the MDA-MB-231 tumors. Anti-EGFR-GN or a combination of anti-EGFR-GN and NIR-PTT may provide additional therapeutic potential for those TNBC patients overexpressing EGFR who do not respond to anti-EGFR antibody treatment.

In the present study, we suggest that NIR-PTT combined with anti-EGFR-GN is an encouraging therapeutic strategy to improve conventional EGFRtargeted therapy for TNBC patients. However, for the clinical use of anti-EGFR-GNs combined with NIR-PTT, nanoparticle size $(5-6 \mathrm{~nm})$ should be reduced in order for the body to more easily clear them [34]. In regards to the clinical use of wIRA, the typical total irradiances are approximately $80-160 \mathrm{~mW} / \mathrm{cm}^{2}$. For the use of wIRA in the treatment of breast cancer, application of wIRA with appropriate therapeutic irradiation intensities and doses should be considered as being safe depending on the size of the irradiated area, tissue temperature, and the amount of subcutaneous soft tissues.

\section{MATERIALS AND METHODS}

\section{GN, anti-EGFR-GN and breast cancer cell lines}

GN and anti-EGFR-GN (10 $\mathrm{nm} \times 40 \mathrm{~nm})$ conjugated with 16 anti-EGFR antibodies per GN were purchased from Nanopartz, Inc. (Loveland, CO, USA). NIR responsive GN and anti-EGFR-GN were synthesized as previously reported [22]. The human breast cancer cell lines were obtained from the Korean Cell Line Bank (Seoul, Korea): estrogen receptor positive (ER+) (MCF7), human epidermal growth factor receptor 2 amplified (HER2+) (BT-474) and TN (Hs578T, HCC-38, HCC1937, MDA-MB-453 and MDA-MB-231). MDA-MB231-Luc cells stably expressing firefly luciferase were established using lentivirus.

\section{Immunocytochemistry}

Cells were fixed in $2 \%$ paraformaldehyde and blocked with $2 \%$ bovine serum albumin. The cells were incubated with anti-EGFR antibody (Cell Signaling Technology, Danvers, MA, USA), followed by incubation with an appropriate secondary antibody. The proteins were visualized with 3,3-diaminobenzidine, and hematoxylin was used as counterstain. The images were acquired using a microscope equipped with a CCD camera (Leica, Wetzlar, Germany).

\section{Transmission electron microscopy (TEM)}

Cells were fixed with $2.5 \%$ glutaraldehyde, treated with $2 \%$ osmium tetroxide in $0.1 \mathrm{mmol} / \mathrm{L}$ cacodylate buffer for $2 \mathrm{~h}$, dehydrated with ethanol (50 to 100\%) and propylene oxide, and embedded in pure Epon resin at $60^{\circ} \mathrm{C}$ for 3 days. Ultrathin sections were cut with glass knives and a Diatome diamond knife (Reichert-Jung, Vienna, Austria) using an ultramicrotome (RMC MTXL; Tucson, AZ, USA), stained with lead citrate and uranyl acetate and observed with a JEM-100 CX transmission electron microscope (JEOL, Tokyo, Japan).

\section{3-(4,5-Dimethylthiazol-2-yl)-2,5-diphenyl tetrazolium bromide (MTT) assay}

MTT solution ( $1 \mathrm{mg} / \mathrm{ml})$ was administrated and cells were incubated for $1 \mathrm{~h}$. At the end of the incubation period, $150 \mu \mathrm{l}$ of dimethyl sulfoxide was added to each well. The amount of formazan crystals formed by the viable cells was determined using a spectrophotometer at $540 \mathrm{~nm}$ (GE Healthcare, Piscataway, NJ, USA).

\section{Flow cytometry}

Flow cytometry was performed using a FACS Calibur flow cytometer (BD Biosciences, San Jose, CA, USA). The cells were incubated with annexin $\mathrm{V}$ and propidium iodide (PI). Cell damage was assessed with flow cytometric evaluation of cells stained by annexin $\mathrm{V}$ and PI, and the data were analyzed using Cell Quest v3.3 software.

\section{Western blot analysis}

Proteins of total cell lysates were separated using SDS-PAGE and transferred to nitrocellulose membranes. 
The membranes were incubated with primary antibodies, followed by incubation with an appropriate secondary antibody. The following primary antibodies were used in this study: anti-EGFR, anti-phospho-AKT, anti-AKT, anti-phospho-ERK1/2, anti-ERK1/2, anti-phosphomTOR, anti-mTOR, anti-cleaved caspase-3 and anti-FAK antibodies (Cell Signaling Technology, Danvers, MA, USA), anti-phospho-FAK and anti- $\beta$-actin antibodies (Sigma, St. Louis, MO, US), anti-HSP70 and anti-HSP90 antibodies (Abcam, Cambridge, MA, USA), and Ki-67 antibody (Santa Cruz Biotechnology, Santa Cruz, CA, USA). The relative intensity of the bands observed by western blotting was analyzed using the Image J program.

\section{Xenograft tumor model}

Female BALB/c nude mice (5-6 weeks old; Orient Bio, Sungnam, Korea) were housed in the animal care facility of the Biomedical Research Institute of Seoul National University Hospital. Animal care and experimental procedures were carried out in accordance with guidelines on the ethical use of animals that were approved by the Institutional Animal Care and Use Committee (IACUC) of Seoul National University Hospital (12-0353-C2A0). $1 \times 10^{6}$ viable cells were injected into the right fat pad of the 4th mammary gland. Tumor volume was measured with digital calipers and US imaging using a modified ellipsoidal formula for volume $\left(\right.$ volume $=1 / 2\left(\right.$ length $\times$ width $\left.\left.^{2}\right)\right)$ [35]. Mice injected with MDA-MB-231-Luc cells were randomly assigned to 4 groups: saline (control) $(n=5)$; anti-EGFR-GN $(n=5)$; anti-EGFR-GN+NIR-PTT $(n=5)$; anti-EGFR $(n=5)$. Mice injected with MCF-7 cells were assigned 2 groups: GN $(\mathrm{n}=5)$; and anti-EGFR-GN $(\mathrm{n}=5)$.

\section{NIR-PTT}

Heat was applied using a wIRA (Hydrosun model 750, Hydrosun Medizintechnik GmbH, Müllheim, Germany) with a $750 \mathrm{~W}$ halogen lamp and a $780 \mathrm{~nm}$ high pass filter, yielding a peak output at $820 \mathrm{~nm}$. In vitro NIRPTT (at $1.5 \mathrm{~W} / \mathrm{cm}^{2}$ for $3 \mathrm{~min}$ ) was performed in cultured cells at $24 \mathrm{~h}$ after treatment with anti-EGFR, GN or antiEGFR-GN. Based on our previous study [22], we decided the NIR-PTT time point at which anti-EGFR-GNs were maximally accumulated into the tumors of mice after intravenous injection of anti-EGFR-GNs using serial follow-up PAI. When the average tumor volume was 150 $200 \mathrm{~mm}^{3}$, a circular mask $1.5 \mathrm{~cm}$ in diameter was placed over the abdomen to confine the radiation to the tumor region and in vivo NIR-PTT (at $1.5 \mathrm{~W} / \mathrm{cm} 2$ for $3 \mathrm{~min}$ ) at 48 $\mathrm{h}$ after intravenous injection of anti-EGFR-GNs (100 nM, final $0.5 \mathrm{pmol} / \mathrm{g}$ mouse) at 3 d-intervals was performed three times. The temperature of culture medium and tumor skin was measured using the non-contact infrared thermometer gun (BENETECH, Shenzhen, China).

\section{US-guided PAI and bioluminescence imaging (BLI)}

US-guided PAI was performed in B mode and PA mode using a preclinical Vevo2100 LAZR imaging system (FUJIFILM VisualSonics Inc., Toronto, Ontario, Canada) equipped with a $40 \mathrm{MHz}$, linear array transducer. The laser was tuned to optical wavelengths from 750 to $850 \mathrm{~nm}$ with a PA signal gain of $40 \mathrm{~dB}$. The relative PA signal amplitude on image slices of tumor was quantified using the post-processing software tools (FUJIFILM VisualSonics Inc., Toronto, Ontario, Canada) [36]. In vivo BLI after intraperitoneal injection of D-luciferin (150 ng/kg, Promega, San Luis Obispo, CA, USA) was conducted on the IVIS luminal II system (Caliper, Hopkinton, MA, USA). The sum of all detected photon counts within tumor was quantified in units of mean photons per second per centimeter squared per steradian $\left(\mathrm{p} / \mathrm{s} / \mathrm{cm}^{2} / \mathrm{sr}\right)$.

\section{Histological analysis}

The tumor, liver, spleen and kidney were removed, fixed with $10 \%$ buffered formalin and embedded in paraffin blocks. Hematoxylin and eosin (H\&E) staining, immune gold-silver staining (Sigma, St. Louis, MO USA) and immunostaining for cleaved caspase-3, Ki-67 and EGFR were performed. Terminal uridine deoxynucleotidyl transferase-mediated dUTP nick end labeling (TUNEL) staining with the ApopTag Peroxidase In Situ Apoptosis Detection Kit (S7100, Millipore Ltd., Darmstadt, Germany) was used to detect apoptotic cells. Histological images of stained tissues were acquired using a microscope (Leica, Wetzlar, Germany) equipped with a CCD camera.

\section{Statistical analyses}

All experiments were expressed as the means \pm standard errors calculated from at least five independent experiments. The ANOVA was performed. A $P$ value less than 0.05 was considered statistically significant.

\section{Abbreviations}

NIR-PTT, Near-infrared photothermal therapy; PAI, Photoacoustic imaging; TNBC, Triple negative breast cancer, GN, Gold nanorod; EGFR, Epidermal growth factor receptor; anti-EGFR-GN, anti-EGFR antibodyconjugated gold nanorod.

\section{CONFLICTS OF INTEREST}

There are no conflicts of interest to disclose. 


\section{GRANT SUPPORT}

This research was supported by the Basic Science Research Program through the National Research Foundation of Korea (NRF) funded by the Ministry of Education, Science and Technology (2015R1D1A1A01059376). M.Z. and T.J. are the awardees of graduate student and postdoctoral fellowships, respectively, funded by Brain Korea 21 Plus (BK21 Plus).

\section{REFERENCES}

1. Kaufmann M, Pusztai L, Biedenkopf Expert Panel Members. Use of standard markers and incorporation of molecular markers into breast cancer therapy: Consensus recommendations from an International Expert Panel. Cancer. 2011;117:1575-1582.

2. Goldhirsch A, Wood WC, Coates AS, Gelber RD, Thurlimann B, Senn HJ, Panel members. Strategies for subtypes--dealing with the diversity of breast cancer: highlights of the St. Gallen International Expert Consensus on the Primary Therapy of Early Breast Cancer 2011. Ann Oncol. 2011;22:1736-1747.

3. Crown J, O'Shaughnessy J, Gullo G. Emerging targeted therapies in triple-negative breast cancer. Ann Oncol. 2012;23:vi56-65.

4. Lehmann BD, Bauer JA, Chen X, Sanders ME, Chakravarthy AB, Shyr Y, Pietenpol JA. Identification of human triple-negative breast cancer subtypes and preclinical models for selection of targeted therapies. J Clin Invest. 2011; 121:2750-2767.

5. Carey LA, Rugo HS, Marcom PK, Mayer EL, Esteva FJ, Ma CX, Liu MC, Storniolo AM, Rimawi MF, Forero-Torres A, Wolff AC, Hobday TJ, Ivanova A, et al. TBCRC 001: randomized phase II study of cetuximab in combination with carboplatin in stage IV triple-negative breast cancer. J Clin Oncol. 2012;30:2615-2623.

6. Baselga J, Arteaga CL. Critical update and emerging trends in epidermal growth factor receptor targeting in cancer. $\mathrm{J}$ Clin Oncol. 2005;23:2445-2459.

7. Wang LV, Hu S. Photoacoustic tomography: in vivo imaging from organelles to organs. Science. 2012; 335:1458-1462.

8. Manohar S, Ungureanu C, Van Leeuwen TG. Gold nanorods as molecular contrast agents in photoacoustic imaging: the promises and the caveats. Contrast Media Mol Imag. 2011;6:389-400.

9. Luke GP, Myers JN, Emelianov SY, Sokolov KV. Sentinel lymph node biopsy revisited: ultrasound-guided photoacoustic detection of micrometastases using molecularly targeted plasmonic nanosensors. Cancer Res. 2014;74:5397-5408.

10. Khlebtsov N, Dykman L. Biodistribution and toxicity of engineered gold nanoparticles: a review of in vitro and in vivo studies. Chem Soc Rev. 2011;40:1647-1671.

11. Gobin AM, Lee MH, Halas NJ, James WD, Drezek RA, West JL. Near-infrared resonant nanoshells for combined optical imaging and photothermal cancer therapy. Nano Letters. 2007;7:1929-1934.

12. Au L, Zheng D, Zhou F, Li ZY, Li X, Xia Y. A quantitative study on the photothermal effect of immuno gold nanocages targeted to breast cancer cells. ACS Nano. 2008; 2:1645-1652.

13. Piao JG, Wang L, Gao F, You YZ, Xiong Y, Yang L. Erythrocyte membrane is an alternative coating to polyethylene glycol for prolonging the circulation lifetime of gold nanocages for photothermal therapy. ACS Nano. 2014;8:10414-10425.

14. Weissleder R, Pittet MJ. Imaging in the era of molecular oncology. Nature. 2008; 452:580-589.

15. Jang B, Park JY, Tung CH, Kim IH, Choi Y. Gold nanorodphotosensitizer complex for near-infrared fluorescence imaging and photodynamic/photothermal therapy in vivo. ACS Nano. 2011;5:1086-1094.

16. Liang C, Diao S, Wang C, Gong H, Liu T, Hong G, Shi $\mathrm{X}$, Dai H, Liu Z. Tumor metastasis inhibition by imagingguided photothermal therapy with single-walled carbon nanotubes. Adv Mater. 2014; 26:5646-5652.

17. Xiao Z, Ji C, Shi J, Pridgen EM, Frieder J, Wu J, Farokhzad OC. DNA self-assembly of targeted nearinfrared-responsive gold nanoparticles for cancer thermo-chemotherapy. Angew Chem Int Ed Engl. 2012;51:11853-11857.

18. Ke H, Yue X, Wang J, Xing S, Zhang Q, Dai Z, Tian J, Wang S, Jin Y. Gold nanoshelled liquid perfluorocarbon nanocapsules for combined dual modal ultrasound/CT imaging and photothermal therapy of cancer. Small. 2014; 10:1220-1227.

19. Shen S, Kong F, Guo X, Wu L, Shen H, Xie M, Wang $\mathrm{X}$, Jin Y, Ge Y. CMCTS stabilized Fe3O4 particles with extremely low toxicity as highly efficient near-infrared photothermal agents for in vivo tumor ablation. Nanoscale. 2013;5:8056-8066.

20. Akhavan O, Ghaderi E. Graphene nanomesh promises extremely efficient in vivo photothermal therapy. Small. 2013;9:3593-3601.

21. Antaris AL, Robinson JT, Yaghi OK, Hong G, Diao S, Luong R, Dai H. Ultra-low doses of chirality sorted $(6,5)$ carbon nanotubes for simultaneous tumor imaging and photothermal therapy. ACS Nano. 2013; 7:3644-3652.

22. Zhang M, Kim HS, Jin T, Yi A, Moon WK. Ultrasoundguided photoacoustic imaging for the selective detection of EGFR-expressing breast cancer and lymph node metastases. Biomed Opt Express. 2016;7:1920-1931.

23. Voduc KD, Cheang MC, Tyldesley S, Gelmon K, Nielsen TO, Kennecke H. Breast cancer subtypes and the risk of local and regional relapse. J Clin Oncol. 2010;28:1684-1691.

24. Sunada H, Magun BE, Mendelsohn J, MacLeod CL. Monoclonal antibody against epidermal growth factor receptor is internalized without stimulating receptor phosphorylation. Proc Natl Acad Sci U S A. 1986;83:3825-3829. 
25. Al-Ejeh F, Shi W, Miranda M, Simpson PT, Vargas AC, Song S, Wiegmans AP, Swarbrick A, Welm AL, Brown MP, Chenevix-Trench G, Lakhani SR, Khanna KK. Treatment of triple-negative breast cancer using antiEGFR-directed radioimmunotherapy combined with radiosensitizing chemotherapy and PARP inhibitor. J Nucl Med. 2013;54:913-921.

26. Ferraro DA, Gaborit N, Maron R, Cohen-Dvashi H, Porat Z, Pareja F, Lavi S, Lindzen M, Ben-Chetrit N, Sela M, Yarden Y. Inhibition of triple-negative breast cancer models by combinations of antibodies to EGFR. Proc Natl Acad Sci U S A. 2013; 110:1815-1820.

27. Jain S, Hirst DG, O'Sullivan JM. Gold nanoparticles as novel agents for cancer therapy. Br J Radiol. 2012;85:101-113.

28. Wu P, Gao Y, Zhang H, Cai C. Aptamer-guided silvergold bimetallic nanostructures with highly active surfaceenhanced Raman scattering for specific detection and nearinfrared photothermal therapy of human breast cancer cells. Anal Chem. 2012;84:7692-7699.

29. Zou L, Wang H, He B, Zeng L, Tan T, Cao H, He X, Zhang Z, Guo S, Li Y. Current Approaches of Photothermal Therapy in Treating Cancer Metastasis with Nanotherapeutics. Theranostics. 2016;6:762-772.

30. Hoffmann G. Principles and working mechanisms of waterfiltered infrared-A (wIRA) in relation to wound healing. GMS Krankenhhyg Interdiszip. 2007; 2:Doc54.
31. Singh M, Harris-Birtill DC, Zhou Y, Gallina ME, Cass AE, Hanna GB, Elson DS. Application of Gold Nanorods for Photothermal Therapy in Ex Vivo Human Oesophagogastric Adenocarcinoma. J Biomed Nanotechnol. 2016;12:481-490.

32. Lim CK, Shin J, Lee YD, Kim J, Oh KS, Yuk SH, Jeong SY, Kwon IC, Kim S. Phthalocyanine-aggregated polymeric nanoparticles as tumor-homing near-infrared absorbers for photothermal therapy of cancer. Theranostics. 2012; 2:871-879.

33. Zhang M, Kim HS, Jin T, Moon WK. Near-infrared photothermal therapy using EGFR-targeted gold nanoparticles increases autophagic cell death in breast cancer. J Photochem Photobiol B. 2017;170:58-64.

34. Choi HS, Liu W, Misra P, Tanaka E, Zimmer JP, Itty Ipe B, Bawendi MG, Frangioni JV. Renal clearance of quantum dots. Nat Biotechnol. 2007;25:1165-1170.

35. Jensen MM, Jorgensen JT, Binderup T, Kjaer A. Tumor volume in subcutaneous mouse xenografts measured by microCT is more accurate and reproducible than determined by $18 \mathrm{~F}-F D G-m i c r o P E T$ or external caliper. BMC Medical Imaging. 2008;8:16.

36. Wang LV, Gao L. Photoacoustic microscopy and computed tomography: from bench to bedside. Annu Rev Biomed Eng. 2014;16:155-185. 